\title{
Phlebotonics for haemorrhoids
}

\author{
Nirmal Pereira, Danae Liolitsa, Satheesh lype, Anna \\ Croxford, Muhhamed Yassin, Lang Peter, Obioha \\ Ukaegbu, Christopher van Issum
}

\section{The independent commentary was written by Sarhan Sydney Saad}

\begin{abstract}
BACKGROUND: Haemorrhoids are variceal dilatations of the anal and perianal venous plexus and often develop secondary to the persistently elevated venous pressure within the haemorrhoidal plexus. Phlebotonics are a heterogenous class of drugs consisting of plant extracts (i.e. flavonoids) and synthetic compounds (i.e. calcium dobesilate). Although their precise mechanism of action has not been fully established, they are known to improve venous tone, stabilize capillary permeability and increase lymphatic drainage. They have been used to treat a variety of conditions including
\end{abstract} chronic venous insufficiency, lymphoedema and haemorrhoids.

OBJECTIVE: The aim of this review was to investigate the efficacy of phlebotonics in alleviating the signs, symptoms and severity of haemorrhoidal disease and verify their effect post-haemorrhoidectomy. METHODS:

Search methods: We searched the Cochrane Central Register of Controlled Trials (CENTRAL) in the Cochrane Library 2011 issue 9, MEDLINE (1950 to September 2011) and EMBASE (1974 to September 2011).

Selection criteria: Only randomized controlled trials evaluating the use of phlebotonics in treating haemorrhoidal disease were used. No crossover or cluster-randomized trials were included for analysis and any trial which had a quasi-random method of allocation was excluded. Data collection and analysis: Two authors independently extracted the data and analyzed the eligibility of the data for inclusion. Disagreements were resolved by meaningful discussion.

MAIN RESULTS: We considered twenty-four studies for inclusion in the final analysis. Twenty of these studies (enrolling a total of 2344 participants) evaluated the use of phlebotonics versus a control intervention. One of these twenty studies evaluated the use of phlebotonics with a medical intervention and another study with rubber band ligation. AUTHORS' CONCLUSIONS: The evidence suggests that there is a potential benefit in using phlebotonics in treating haemorrhoidal disease as well as a benefit in alleviating post-haemorrhoidectomy symptoms. Outcomes such as bleeding and overall symptom improvement show a statistically significant beneficial effect and there were few concerns regarding their overall safety from the evidence presented in the clinical trials.Only randomized controlled trials evaluating the use of phlebotonics in treating haemorrhoidal disease were used. No cross-over or cluster-randomized trials were included for analysis and any trial which had a quasi-random method of allocation was excluded.

This is the abstract of a Cochrane Review published in the Cochrane Database of Systematic Reviews (CDSR) 2013, issue 5, Art. No. CD004322. DOI: 10.1002/14651858.CD004322.pub8 (http://onlinelibrary.wiley.com/doi/10.1002/14651858.CD004322.pub3/abstract). For full citation and authors details see reference 1

The full text is freely available from: http://cochrane.bvsalud.org/ cochrane/main.php?lib=COC\&searchExp=Phlebotonics\%20and\%20 for\%20and\%20haemorrhoids\&lang=pt (this link may be temporary)

\section{REFERENCE}

1. Perera $N$, Liolitsa $D$, lype $S$, et al. Phlebotonics for haemorrhoids. Cochrane Database Syst Rev. 2012;8:CD004322.

\section{COMMENTS}

Hemorrhoidal disease is responsible for frequent seeking of medical attention, due to a variable range of symptoms and signs. It is often treated through use of supplementary fiber in the diet and local medication. Use of phlebotonics for treating hemorrhoids has always been a matter of discussion because the evidence in the literature has not indicated that they should be used routinely. Their action seems to be based on increasing the strength and tone of the vessel wall, thereby improving lymphatic drainage and normalizing capillary permeability.

The aim of this systematic review was to observe the action of these substances in relieving the symptoms of hemorrhoids and their effect during the postoperative period following hemorrhoidectomy. Twentyfour prospective randomized studies that evaluated the use of phlebotonics in hemorrhoidal disease were selected using strict criteria. These studies compared use of phlebotonics with control interventions or even elastic ligation.

The results, based on statistically significant numbers, showed that these substances are safe to use, with a low rate of side effects, and that they provide improvements in symptoms with regard to bleeding and postoperative pain, and in other symptoms such as itching.

Thus, this systematic review has obtained the evidence that is needed for phlebotonics to be used in medical treatments for hemorrhoidal disease.

Sarhan Sydney Saad. MD, PhD. Associate Full Professor and Head of the Coloproctology Group, Discipline of Gastrointestinal Surgery, Escola Paulista de Medicina - Universidade Federal de São Paulo (EPMUnifesp), São Paulo (SP), Brazil. 